\title{
A reabilitação psicossocial como estratégia de cuidado: percepções e práticas desenvolvidas por trabalhadores de um serviço de saúde mental
}

\section{The psychosocial rehabilitation as a care strategy: perceptions and practices developed by workers of a mental health service}

\author{
Mara Cristina Ribeiro ${ }^{1}$, Waldez Cavalcante Bezerra ${ }^{2}$
}

http://dx.doi.org/10.11606/issn.2238-6149.v26i3p301-308

Ribeiro MC, Bezerra WC. A reabilitação psicossocial como estratégia de cuidado: percepções e práticas desenvolvidas por trabalhadores de um serviço de saúde mental. Rev Ter Ocup Univ São Paulo. 2015 set.-dez.;26(3):301-8.

RESUMO: As ações desenvolvidas nos Centros de Atenção Psicossocial (CAPS) defrontam-se com impasses clínicos e operacionais quando avaliadas sob a perspectiva da Reabilitação Psicossocial. O estudo, desenvolvido em um CAPS da cidade de São Paulo, visou conhecer como o conceito da Reabilitação Psicossocial é assimilado por seus trabalhadores, como estes a relacionam às suas práticas e como a percebem na instituição em que estão inseridos. A abordagem metodológica foi qualitativa. Participaram do estudo oito trabalhadores. Para a produção dos dados foi utilizada a entrevista semiestruturada e para a análise a técnica de Análise Temática. Verificou-se que a condução prática das ações do cuidado em saúde mental deve estar embasada pelo entendimento do que é o processo de Reabilitação Psicossocial. Também se observou que os trabalhadores percebem de forma crítica as condutas institucionais que priorizam intervenções dentro do serviço e pouco articuladas aos contextos externos. O estudo sugere que trabalhar com a estratégia da Reabilitação Psicossocial exige flexibilidade no enfrentamento de diferentes desafios, garantindo a presença da coletividade no processo. Também aponta para a necessidade de elaboração de instrumentos de monitoramento e avaliação dos serviços.

DESCRITORES: Saúde mental; Serviços de saúde mental; Centros de reabilitação; Pessoal de saúde/psicologia.
Ribeiro MC, Bezerra WC. The psychosocial rehabilitation as a care strategy: perceptions and practices developed by workers of a mental health service. Rev Ter Ocup Univ São Paulo. 2015 Sept.-Dec.;26(3):301-8.

ABSTRACT: The actions developed in the Psychosocial Care Centers are confronted with clinical and operational impasses when assessed from the perspective of Psychosocial Rehabilitation. The study, conducted in a CAPS in São Paulo aimed to know how the concept of Psychosocial Rehabilitation is assimilated by its workers, as they relate it to their practices and how they perceive the institution to which they belong to. The methodological approach was qualitative. Study participants were 08 professional workers. For the production of data semi-structured interview was used, and for the analysis the Thematic Analysis technique was used. It was found that the practical conduct of care actions in mental health must be grounded by understanding concerning the nature of the Psychosocial Rehabilitation process. It was also observed that workers perceive critically institutional conducts that prioritize interventions within the service and are little articulated in relation to external contexts. The study suggests that working with the Psychosocial Rehabilitation strategy requires flexibility in facing different challenges, ensuring the community's presence in the process. It also points to the need of creating instruments to monitor and evaluate the services.

KEYWORDS: Mental health; Mental health services; Rehabilitation centers; Health personnel/psychology.

Artigo é parte da dissertação da primeira autora, Programa de Pós-Graduação em Enfermagem Psiquiátrica, Escola de Enfermagem da Universidade de São Paulo.

1.Universidade Estadual de Ciências da Saúde de Alagoas (UNCISAL), Curso de Terapia Ocupacional. E-mail: maracrisribeiro@gmail. com.

2. Universidade Estadual de Ciências da Saúde de Alagoas (UNCISAL), Curso de Terapia Ocupacional. E-mail: waldezto@yahoo.com.br. Endereço para correspondência: Mara Cristina Ribeiro. Universidade Estadual de Ciências da Saúde de Alagoas. Centro de Ciências da Saúde. Curso de Terapia Ocupacional. Rua Jorge de Lima, 113 - Trapiche da Barra, Maceió, AL. CEP: 57010-382. E-mail: maracrisribeiro@ gmail.com 


\section{INTRODUÇÃO}

s experiências de transformação da
assistência psiquiátrica brasileira emergiram
ao final da década de 1980 e início de 1990, fruto de reivindicações advindas dos movimentos sociais e dos trabalhadores no final dos anos 1970, com a redemocratização do país. Assim, na cidade de São Paulo surgiu o primeiro Centro de Atenção Psicossocial (CAPS) em 1987 e, em Santos, após a intervenção municipal na Casa de Saúde Anchieta (hospital psiquiátrico privado), foi inaugurado o primeiro Núcleo de Atenção Psicossocial (NAPS) em 1989¹.

O Ministério da Saúde, sob a influência do Movimento de Reforma Psiquiátrica e das experiências em São Paulo e Santos, assumiu a gestão do novo modelo assistencial e propôs mecanismos para viabilizá-lo: a Portaria 189/91 alterou o financiamento das ações e serviços de saúde mental e possibilitou a implantação de novos serviços (CAPS/NAPS, hospital-dia e unidades psiquiátricas em hospitais gerais). São marcos desse processo: a Portaria 224/92 que regulamentou o funcionamento dos serviços de saúde mental e apoiou a regulação de serviços hospitalares, a Lei 10.216 que redirecionou o modelo assistencial em saúde mental e, finalmente, a Portaria 336/02 que ampliou a abrangência dos serviços e criou mecanismos de financiamento próprios, para a rede de $\mathrm{CAPS}^{2}$.

Ao longo dos anos seguintes, foram criados em todo o país, novos serviços de saúde mental com propostas de tratamentos que consideram a singularidade e as condições concretas das pessoas que buscam cuidados.

Neste contexto, a loucura passa a ser percebida em sua complexidade e não mais pela objetivação da psiquiatria; a cidadania passa a ser um dos grandes princípios considerados, ampliando-se os movimentos sociais envolvidos nesse processo, como as associações de familiares e usuários, além de cooperativas e eventos com o objetivo de possibilitar a construção de novas formas de convivência com a sociedade ${ }^{3}$.

Os CAPS emergem como modelo de serviços, com o objetivo de atender pessoas com transtorno mental severo e persistente, oferecer cuidados na perspectiva da clínica ampliada e da reabilitação psicossocial, sob a lógica da territorialidade. Neles o cuidado deve estar alinhado de forma integral e intensiva e oferecer respostas às diversas necessidades apresentadas pelos usuários em suas vidas cotidianas $^{4}$.

Compreende-se aqui reabilitação psicossocial, na perspectiva de Saraceno ${ }^{5,6}$, como processo de reconstrução, de exercício de cidadania e contratualidade nos cenários cotidianos de vida dos usuários dos serviços de saúde mental. Neste sentindo, não é coerente com essa perspectiva o emprego de medidas reabilitativas isoladas dos contextos sociais e de significação dos usuários (sejam eles concretos ou simbólicos), bem como de ações que não permitam a integração interna e externa dos serviços, garantindo a permeabilidade destes, e a flexibilidade na organização dos processos de trabalho ${ }^{5,6}$.

Embora, conceitualmente, os CAPS representem um dos importantes operadores das práticas inovadoras de transformação da vida das pessoas com sofrimento psíquico e a reabilitação psicossocial a estratégia utilizada para o alcance desse objetivo, é preciso aprofundar o conhecimento das práticas exercidas nesses serviços.

Nos últimos anos, a implantação de CAPS em todo o país, defronta-se com grandes impasses clínicos e operacionais, pois mesmo no interior dos serviços de vanguarda é possível encontrar velhas formas de psiquiatrização, que aprisionam o indivíduo, isolando-o do meio social, reforçando o caráter excludente ${ }^{7}$.

Nesse sentido, estudos ${ }^{8,9}$ vêm apontando a necessidade de articulação das ações técnicas exercidas no bojo do cuidado clínico com movimentos de reinserção dos sujeitos ao seu território, à sua família e comunidade.

Portanto, as atuais estratégias de atenção em saúde mental desencadeiam nas equipes multiprofissionais dos CAPS a necessidade de reestruturação frente às novas propostas de intervenção. Assim, este trabalho se propõe a refletir sobre essas inquietações - tendo como eixo fundamental a reabilitação psicossocial.

Acredita-se que o conhecimento de como o conceito da reabilitação psicossocial está sendo assimilados pelos profissionais de saúde mental, como estes a relacionam às suas práticas e como a percebem na instituição na qual estão inseridos - objetivos deste estudo - pode trazer subsídios para sua melhor aplicação no cuidado às pessoas que sofrem com transtornos mentais, bem como problematizar esta questão, diferenciando o que é o conhecimento teórico e o que é o exercício prático da reabilitação psicossocial no cotidiano dos serviços.

\section{MÉTODOS}

Para viabilizar o estudo todos os critérios éticos foram assumidos e a pesquisa foi previamente aprovada pelo Comitê de Ética em Pesquisa da Escola de Enfermagem da Universidade de São Paulo sob o número 311/2003.

A metodologia escolhida para o desenvolvimento da pesquisa foi a qualitativa. Foram entrevistados trabalhadores de um CAPSII da cidade de São Paulo, inserido em uma 
rede de cuidado em saúde mental, composta por leitos psiquiátricos em hospital geral e Centro de Convivência e Cooperativa, e integrado a programa de ensino articulado com Universidades.

Os trabalhadores do serviço foram informados da realização da pesquisa em uma reunião de rotina da instituição, em que participavam tanto profissionais com formação de nível superior quanto de nível médio. Foram apresentados os objetivos do estudo e todos foram convidados a participar. Foi realizado contato e agendado dia e horário próprios para a realização da entrevista de forma individual com aqueles que indicaram interesse e respondiam aos seguintes critérios de inclusão: trabalhar no serviço por um período igual ou superior a seis meses e estar envolvido diretamente com atividades (individuais e/ ou grupais) oferecidas para os usuários do CAPS.

Utilizou-se como instrumento para a produção dos dados a entrevista semiestruturada, a partir de um único roteiro, elaborado pelos pesquisadores, que permitiu a abordagem das questões consideradas importantes para a temática. As entrevistas foram gravadas com a permissão dos participantes.

Para a análise dos dados foi utilizada a análise temática. Todas as entrevistas foram transcritas e, a partir disso, foi realizada a etapa de leitura flutuante. Depois, as falas foram codificadas e separadas por meio do processo de categorização. Esse processo de tratamento dos resultados permitiu a construção de inferências e a interpretação das falas. Seguiu-se, portanto, as diferentes fases da análise: pré-análise, exploração do material e tratamento dos resultados ${ }^{10}$.

\section{APRESENTAÇÃO E DISCUSSÃO DOS RESULTADOS}

Participaram do estudo oito profissionais do serviço que estavam diretamente envolvidos na assistência aos usuários, sendo cinco com formação de nível superior (dois terapeutas ocupacionais, dois psicólogos e um médico psiquiatra) e três com formação de nível técnico (três auxiliares de enfermagem), totalizando oito entrevistas. Todos os participantes eram do sexo feminino e a média de idade foi de 42 anos e meio, com mínimo de 31 anos e máximo de 62 anos.

Com relação à caracterização dos trabalhadores entrevistados segundo o tempo de serviço, o estudo revelou que o tempo médio de trabalho em saúde mental foi de 11 anos e 3 meses e o tempo de trabalho específico no CAPSII em que a pesquisa foi realizada foi de cinco anos e dois meses.

As três categorias temáticas que emergiram da análise das entrevistas foram: a concepção da reabilitação psicossocial; as práticas de reabilitação psicossocial; e, os desafios no campo da reabilitação psicossocial. A seguir, apresentamos cada uma delas com suas possíveis inferências e interpretações.

\section{A concepção da reabilitação psicossocial}

O resgate da autonomia possível e a ampliação das possibilidades de trocas sociais sejam elas afetivas, materiais ou simbólicas, se constituíram, para os entrevistados, a marca do que é considerado o processo de reabilitação psicossocial.

"Penso que é um processo que visa favorecer a inclusão das pessoas portadoras de transtorno mental nas coisas da comunidade, favorecer a convivência, o estar junto, preparar o sujeito para que ele possa estar conseguindo participar das coisas da comunidade" (P.6).

"[...] um simples resgatar de escrever, de fazer uma conta, de poder conversar, de poder até ir num cinema, fazer as coisas mais simples, se elas conseguirem isso, eu acho que isso é uma reabilitação psicossocial” (P.2).

A reabilitação psicossocial se define enquanto conjunto de estratégias destinadas a aumentar as oportunidades de troca de recursos e afetos, processo esse que necessariamente implica na abertura de espaços de negociação para o usuário, para a sua família, para a comunidade circundante e para os serviços implicados em seu tratamento. Desta forma, seu conceito pode ser ampliado como processo que permite e estimula a inserção social e o desenvolvimento de potencialidades que ao longo do convívio com o sofrimento psíquico ficaram encobertas ${ }^{11}$.

Alguns cenários de exercício desse processo foram citados pelos participantes da pesquisa:

"Eu vejo a reabilitação psicossocial no âmbito da vida, da vida social, familiar, da vida total do indivíduo, ele poder trabalhar, ele poder se divertir e ele poder conviver com as pessoas novamente" (P.2).

"Eu penso na reabilitação como um trabalho para o paciente voltar para o mercado, para conseguir um trabalho, para ter renda" (P.3).

Os trechos revelam que a ideia e prática desenvolvidas estão de acordo com conceitos teóricos que balizam a 
reabilitação psicossocial, mesmo que estes não sejam referenciados, confirmando os estudos que apontam que a construção do significado da reabilitação psicossocial é feita na experiência cotidiana ${ }^{9}$.

No entanto, algumas concepções trazidas chamam a atenção por evidenciarem uma visão reducionista sobre os objetivos que se pretende com a reabilitação psicossocial:

"acredito que é uma recuperação, não sei a quantos por cento, mas que é uma recuperação é, porque é uma recondução ao mais normal possivel da pessoa, às vezes não é tudo aquilo que ela tinha, mas é o mais normal que possa parecer" (P.2).

"vai servir para eles estarem se inserindo nesse mundo que a gente diz que é normal, pois a gente mesmo os considera anormais, para mim a reabilitação é eles ficarem dentro, estar junto, dentro do conceito de sociedade que a gente vive, não ficar à margem" (P5).

"tem paciente que está tendo a primeira crise ou tem pouco tempo de doença mental, mas ele já está fora do contexto de normalidade" (P.5).

Interessante detectar que a questão da normalidade, ou melhor, dizendo, do retorno do estado normal, fez parte de várias falas, colocando-se dentro dos objetivos a serem alcançados por meio da reabilitação psicossocial.

Canguilhem ${ }^{12}$, em estudo sobre a conceituação do normal e do patológico, traz o conceito de normatividade, que seria a capacidade de instaurar novas normas e não o estado de conformidade perante as normas estabelecidas.

Aguiar $^{13}$, ao analisar as proposições de Canguilhem ${ }^{12}$, afirma que ser normativo seria ter a capacidade de criar padrões para si sempre que necessário e não estar dentro dos padrões esperados. Desta forma, o que caracterizaria um estado patológico seria a diminuição da normatividade, isto é, quando a capacidade de enfrentar os desafios que o meio ambiente impõe estivesse diminuída.

A partir dessa perspectiva pode-se afirmar que tratar em saúde mental de acordo com as proposições da reabilitação psicossocial não deve ser normalizar, mas sim, utilizar-se de todos os meios possíveis para interromper ou amenizar o processo que restringe a normatividade das pessoas $^{13}$.

Para que isso seja possível, é preciso apontar que toda prática cuidadora deve estar vinculada a uma grade conceitual, e caminhar na construção de conceitos norteadores, mesmo que obtida muitas vezes por meio de referenciais próprios, construídos no fazer cotidiano.
Desta forma, a ideia de uma construção teórica não se contrapõe à ideia do exercício da prática, mas sim, pode ser dela resultante. Saraceno ${ }^{14}$ indica que a prática da reabilitação psicossocial está à espera de sua construção teórica e aponta que esse fato não deve gerar pressa, pois ao querermos acelerar esse processo podemos incorrer no risco de construirmos teorias desarticuladas da uma prática possível.

No entanto, algumas inquietações nesse campo são percebidas na fala dos entrevistados:

“[...] hoje eu vou percebendo algum detalhamento dentro desse processo porque eu acho que têm algumas discussões mais aprofundadas, do que é a reabilitação, do sentido simbólico dela, de qual engate que ela faz no mundo, que isso não é uma coisa descolada das propostas de trabalho teóricas que existem, que isso é uma fundamentação teórica" (P.1).

“[...] não acho que nós estamos no começo dessa história, acho até que muitas pessoas promovem reabilitação psicossocial, têm uma visão muito ampliada do trabalho, mas não sabem o que estão fazendo, não fazem junção, acho que tem, vamos dizer, produções de coisas interessantíssimas aqui e muitas vezes descoladas de um pensamento e aí, mal aproveitadas" (P.7).

Compreende-se, a partir dos trechos apresentados, que existe a necessidade de uma condução prática fundamentada pelo embasamento teórico e, dessa forma, apropriada de um entendimento do que é o processo de Reabilitação Psicossocial e de como ele pode se articular nas ações do cuidado em saúde mental.

Além disso, outras dificuldades podem ser identificadas nas entrevistas:

"Eu percebo que a área clínica é mais protegida, porque você tem um referencial muito mais bem elaborado, muito mais longo, uma história teórica muito longa que te dá embasamento para atuar, já na área de reabilitação acho que as ideias são relativamente muito mais novas e põe as pessoas que estão trabalhando nela muito mais em xeque, porque é tudo muito experimental, as pessoas se veem fora de seu papel profissional tradicional" (P.1).

"Acho que isso é um desafio da nossa prática, acho que nunca vai ter uma resposta pronta, então eu penso que é um processo que está sendo construído aqui nesse CAPS, eu acho que em todos e a gente está tentando ver isso, na verdade, conforme a necessidade de cada paciente, porque não dá pra pensar a reabilitação psicossocial como um pacote fechado, onde a gente vai oferecer coisas fechadas, não dá, porque os pacientes são muito complexos, a doença mental é muito complexa, envolve 
várias coisas, coisas sociais, a gente lida com coisas sociais, coisas econômicas, coisas emocionais, coisas familiares, são várias coisas que estão envolvidas" (P.6).

A reabilitação psicossocial provoca esse questionamento do que se faz, retira os profissionais do lugar confortável de uma clínica restrita - que se entende como a relação de olhar para a doença - e os coloca no campo de uma ação terapêutica ampliada - em que a relação se dá na escuta qualificada do sujeito e daquilo que ele refere como importante para o seu tratamento, para suas relações e trocas sociais, enfim, para a sua vida.

A clínica tradicional tende a transformar o paciente em um objeto inerte, lidando apenas com a doença; a clínica ampliada se contrapõe a essa posição, lidando com a dimensão social e subjetiva dos sujeitos, valorizando o poder terapêutico da escuta e da palavra, na construção e ampliação do seu grau de autonomia ${ }^{15}$.

Portanto, o conceito de reabilitação psicossocial deve estar alinhado às práticas exercidas da clínica ampliada, daí a complexidade ser um conceito fundamental nesse processo, pois seu entendimento impõe o constante repensar dos papéis assumidos pelos profissionais da reabilitação, pondo em cena e problematizando o lugar que o cuidado em saúde mental deve estar: um lugar de conflito, de confronto e contradição. Lugar também de constantes mudanças, pois esta complexidade se refere ao cuidado ofertado para sujeitos, pessoas dentro da coletividade ${ }^{3}$.

\section{As práticas de reabilitação psicossocial}

O pensar e repensar as práticas, perceber a reabilitação como um processo, identificar quais os instrumentos utilizados e como estes se conectam com a reabilitação, vão formando o conjunto de práticas que são percebidas pelos profissionais entrevistados.

"[...] eu comecei a pensar essa questão da reabilitação na prática, com os pacientes, eu fui vendo que reabilitação era algo, entre aspas, natural, era um desdobramento do tratamento mais humanizado" (P.6).

"[...] eu só consigo pensar agora em reabilitação psicossocial quando eu penso no que promove e não no que a gente faz para promover, porque o que a gente faz para promover, pode ser psicoterapia, pode ser oficina, pode ser passear, eu acho que tem que ser uma ação que promova isso, e às vezes é simplesmente um atendimento, a pessoa vem, muda uma postura, vai lá, promove uma mudança. Acho que é a promoção de mudanças com perspectiva de qualidade" (P.7).
Várias estratégias são citadas como facilitadoras do processo de reabilitação psicossocial:

"[...] quando a gente oferece um tratamento que passa pelo eixo da medicação, das psicoterapias e também de oficinas, a gente está cuidando dessas coisas, está tentando favorecer que retorne, que a pessoa possa resgatar a cidadania dela" (P.6).

"A oficina tem um viés importante com a reabilitação nessa coisa da troca, da convivência; são pacientes com muitos anos de doença e acho que ali é um campo onde se possibilita muito essa troca, a troca de afeto, a troca de saber, troca de experiência, troca de vida, enfim, vários tipos de troca, que eu acho que isso é uma coisa da reabilitação, poder ter esse poder de trocar, de falar de si, de tentar fazer um eixo com a vida fora da instituição" (P.8).

Nota-se, de acordo com os entrevistados, uma tendência a considerar as oficinas terapêuticas como o espaço mais bem definido para a sua realização, por se constituir um espaço que oportuniza trocas. $\mathrm{O}$ dispositivo chamado oficina tem sido utilizado para designar um amplo espectro de experiências terapêuticas e extra-terapêuticas que remetem à ideia de produção e desta para a ideia de produção de subjetividade, pois são nesses espaços que se compõem e se experimentam novas formas de relacionamento, novos espaços existenciais e de expressão ${ }^{16}$.

Pesquisas desenvolvidas em outros CAPS, têm considerado o espaço grupal e as oficinas como espaços ricos, potencialmente criativos e que operam na direção da reabilitação psicossocial ${ }^{17-19}$.

Portanto, elas não devem ser utilizadas com o objetivo de ocupação ou exigências estéticas ou de produtividade, muito menos como agrupamento de pessoas sem objetivos claros do porquê se está ali e o quê se pretende com isso. Elas devem ser entendidas como dispositivos clínicos que têm em sua essência a referência de articulação da dimensão sociopolítica com a dimensão da subjetividade e transversalmente são acompanhadas por uma dimensão ética que as regulam operacionalmente ${ }^{20}$.

Essas dimensões vinculadas ao compromisso de seus trabalhadores com as proposições da reabilitação psicossocial é que podem determinar se esta estratégia tem o potencial criador de transformar concretamente os sujeitos envolvidos, oferecendo-lhes espaços reais de trocas ou se ela é simplesmente mais um espaço regulador, que molda seus participantes segundo os desejos dos profissionais responsáveis ou as necessidades institucionais - a história da psiquiatria tem exemplos incontáveis desse último uso. 
O resgate da cidadania também foi apontado como resultado esperado nas práticas desenvolvidas pelo processo de reabilitação psicossocial. No entanto, é preciso entender que essa cidadania reivindicada pelas novas perspectivas de cuidado em saúde mental não tem correspondência única com a cidadania perseguida pelos movimentos sociais - os quais buscam a mera concessão de direitos. A cidadania referenciada por Saraceno ${ }^{6}$, entre tantos outros articuladores técnicos-políticos envolvidos com o processo de construção teórico-prática da Reabilitação Psicossocial, faz parte do conjunto do que Santos ${ }^{21}$ classifica como Novos Movimentos Sociais (NMSs) e incide na afirmação da subjetividade perante esta cidadania: suas formas de opressão e exclusão exigem uma reconversão global dos processos de socialização e de inculcação cultural e dos modelos de desenvolvimento.

Entendendo, assim, que o cuidado em saúde mental organiza-se em novos exercícios e novas formas de cidadania e construção de direitos na coletividade, os autores acima citados apontam ser por meio do exercício da cidadania que se dá a (re)condução das relações e direitos sociais que ao longo da história de aprisionamento imposto aos doentes mentais foram-lhes arrancados. A reabilitação psicossocial, neste caso, pode ser uma das estratégias para alcançar essa (re)condução.

\section{Os desafios no campo da reabilitação psicossocial}

Os profissionais e os serviços implicados no cuidado em saúde mental dentro da proposição da reabilitação psicossocial elaboram e desenvolvem suas práticas dentro de um cotidiano que convida a diferentes desafios: a necessidade da articulação entre o fazer técnico e o fazer político para o enfrentamento da exclusão dos sujeitos assistidos e da própria saúde mental, que parece sempre estar fora das prioridades dos órgãos que estabelecem as diretrizes para o funcionamento das práticas e construção dos serviços de saúde.

$\mathrm{Na}$ fala dos trabalhadores, por exemplo, evidenciase um sentimento de que o trabalho necessário para a efetivação das mudanças parece estar na boa vontade dos profissionais e não nas condutas e diretrizes governamentais.

\footnotetext{
"Me parece que independente da política de saúde mental no municipio, que atualmente é absolutamente ausente, a unidade vai se refazendo, propondo novas coisas, mas que vem pela formação dos profissionais, não vem de fora, se a gente tivesse seguindo a política do municipio a gente estava perdida porque ela não foi colocada claramente até agora" (P.1).
}

\begin{abstract}
"[...] isso é um trabalho muito simbólico, porque as pessoas tentam fazer, a gente não tem recurso, não tem verba, não tem como você ensinar uma profissão para uma pessoa sem ter recursos verdadeiros, acho que a gente fica patinando, fica tentando fazer, tem o grupo de bijuteria, de costura, que eu acho que podem até fazer isso de verdade, mas que eu acho que a gente ainda está muito preso, fica muito dentro da unidade, não consegue por isso pra fora, mas eu acho que falta muito recurso, muita coisa de curso de verdade, que é pra fazer isso de verdade, acho que é só uma tentativa” (P.3).
\end{abstract}

Estes apontamentos nos levam a pensar sobre a dupla natureza dos CAPS e de seus trabalhadores: estes têm uma função terapêutica voltada para a dinâmica subjetiva do sofrimento, mas também se constituem como formuladores e ordenadores das ações em saúde mental, com preocupações voltadas para a integralidade dessas ações, articulação de recursos, estruturação do sistema ${ }^{22}$.

A questão é como fazer a integração desses papéis ou como assumir a interface clínico-política para dar sentido às suas ações?

Assumir uma posição de abertura para experimentar diferentes práticas, aliada à adoção de uma atitude crítica permanente, bem como a aceitação de modelos que ainda buscam embasamentos teóricos, podem ajudar os profissionais a se perceberem como construtores também desse novo paradigma e, com isso, ao invés de sentirem-se afastados desse processo, apropriarem-se dele na construção de uma nova identidade profissional: a de reabilitadores.

A questão do lugar do exercício da reabilitação também é trazida pelos entrevistados como uma crítica às condutas da instituição que priorizam, muitas vezes, intervenções apenas dentro do serviço, fazendo poucas pontes com a comunidade, a família, entre outros:

\footnotetext{
"acho que tem que ter esse gancho, acho que a oficina não tem que ser o fim dela aqui no Caps, acho que ela tem que ser uma porta de entrada pra abrir possibilidades de saída e da pessoa poder criar laços fora” (P.5).

"dentro da instituição a gente consegue trabalhar a convivencia, estimular a realizar atividades, melhorar um pouco tudo isso (...) mas a gente deveria pensar um pouco mais nessa questão voltada para o social, pra comunidade, como é que ele poderia estar interagindo fora daqui" (P.8).
}

A permeabilização do serviço a saberes e recursos circunstantes, como dito anteriormente, é um diferencial determinante para avaliar se ele está integrado externamente ou não ${ }^{11}$. Nesse sentido, a comunidade deve estar presente 
como fonte inexaurível de recursos existentes e potenciais, tanto humanos quanto materiais, o que inclui a família do usuário como parte dessa comunidade em que o serviço deve relacionar-se permanentemente.

Muitas vezes o que ocorre dentro dos serviços é a institucionalização dos profissionais, que apresentam dificuldade em abandonar o campo da prática institucional conhecida rumo ao desafio de uma prática inovadora, muitas vezes incerta, e aparentemente ou potencialmente menos segura.

No entanto, para esses profissionais, inseridos nos serviços substitutivos, é premente a compreensão de que não é possível a desinstitucionalização de seus usuários sem a presença da instituição na comunidade. Dessa forma é preciso compreender que a estratégia de sustentabilidade dos serviços substitutivos passa pela inserção da instituição junto à comunidade, distinguindo-a enquanto uma realidade complexa, que exprime interesses contrastantes e que, por isso, cabe aos profissionais o papel de interlocutores de seus usuários, de forma a gerarem alianças e conflitos contínuos dentro desse território 6 .

Importante pontuar que essa interlocução deve ser temporária, os profissionais devem emprestar o seu poder contratual na medida em que os usuários apresentem dificuldades na interação com o campo social, porém, o objetivo dessa interlocução é favorecer a habilidade dos sujeitos para fazerem essa interação de forma cada vez mais autônoma.

\section{CONSIDERAÇÕES FINAIS}

O estudo sugere que a Reabilitação Psicossocial exige dos trabalhadores flexibilidade em suas ações e uma nova postura frente aos usuários, em que os objetivos a serem alcançados devem estar ligados à singularidade de cada sujeito, respeitando seus desejos e investindo em suas potencialidades reais.

Sua prática tem sido construída cotidianamente no exercício do cuidado em saúde mental e, apesar de sua elaboração teórica ainda ser frágil, este fato não impede a sua execução, mas evidencia a necessidade de maior embasamento para que as ações possam ter direcionamentos mais claros dentro das equipes, dos serviços e das políticas públicas.

Importante entender que a compreensão da complexidade da ação em saúde mental nos serviços é necessária para instigar discussões, inquietações e desafiar aqueles que se colocam como promotores da Reabilitação Psicossocial, sustentados por um eixo ético, teórico, clínico e político que não só admite, mas requer que as incertezas sejam parte do trabalho, garantindo a busca contínua de respostas que vão sendo transformadas por meio das diferenças de cada sujeito assistido.

Desta forma, os profissionais da saúde mental, implicados nas novas ações da atenção psicossocial, devem ser capazes de enfrentar seus diferentes desafios, garantindo a presença da coletividade no processo de Reabilitação Psicossocial.

Por fim, é preciso apontar que, apesar de o estudo ser referente a apenas um serviço, sabe-se que esta realidade se apresenta de forma semelhante em outros locais. Portanto, indica-se a necessidade de elaboração de instrumentos sistemáticos de monitoramento e avaliação que facilitem a produção de informações sobre o funcionamento dos CAPS, possibilitando às equipes a criação de estratégias técnicas e políticas para o enfrentamento de problemáticas específicas, assim como o planejamento de arranjos operativos que permitam superar os desafios cotidianos dos serviços e concretizar as proposições da Reabilitação Psicossocial.

\section{REFERÊNCIAS}

1. Devera D, Costa-Rosa A. Marcos históricos da reforma psiquiátrica brasileira: transformações na legislação, na ideologia e na práxis. Rev Psicol UNESP. 2008;6(1) [citado 26 nov. 2014]. Disponível em: http://www2.assis.unesp.br/ revpsico/index.php/revista/article/view/46/88.
2. Brasil. Ministério da Saúde. Secretaria-Executiva. Secretaria de Atenção à Saúde. Legislação em saúde mental: 19902004. 4a ed. Brasília (DF); 2004. Disponível em: http:// bvsms.saude.gov.br/bvs/publicacoes/legislacao_saude_ mental_1990_2004_5ed.pdf. 
3. Ribeiro MC, Machado AL. A terapia ocupacional e as novas formas do cuidar em saúde mental. Rev Ter Ocup Univ São Paulo. 2008;19(2):72-5. Disponível em: http://www.revistas. usp.br/rto/article/view/14031.

4. Ribeiro MC. Os Centros de Atenção Psicossocial como espaços promotores de vida: relatos da desinstitucionalização em Alagoas. Rev Ter Ocup Univ São Paulo. 2013;24(3):17482. Disponível em: http://www.revistas.usp.br/rto/article/ view/64900/pdf_24.

5. Saraceno B. Reabilitação psicossocial: uma estratégia para a passagem do milênio. In: Pitta AMF, organizadora. Reabilitação psicossocial no Brasil. São Paulo: Hucitec; 1996. p.13-8.

6. Saraceno B. Libertando identidades: da reabilitação psicossocial à cidadania possível. 2a ed. Rio de Janeiro: Te Corá/Instituto Franco Basaglia; 2001.

7. Pinto ATM, Ferreira AAL. Problematizando a reforma psiquiátrica brasileira: a genealogia da reabilitação psicossocial. Psicol Estudo (Maringá). 2010;15(1):27-34. http://dx.doi.org/10.1590/S1413-73722010000100004.

8. Mielke FB, Kantorski LP, Rosa Jardim VM, Olschowsky A, Machado MS. O cuidado em saúde mental no CAPS no entendimento dos profissionais. Ciên Saúde Coletiva. 2009;14(1):159-64. http://dx.doi.org/10.1590/S141381232009000100021 .

9. Jorge MSB, Randemark NFR, Queiroz MVO1, Ruiz EM. Reabilitação psicossocial: visão da equipe de saúde mental. Rev Bras Enferm. 2006;59(6):734-9. http://dx.doi. org/10.1590/S0034-71672006000600003.

10. Bardin L. Análise de conteúdo. Lisboa: Ed 70; 2011.

11. Lussi IAO, Pereira MAO, Pereira Junior A. A proposta de reabilitação psicossocial de Saraceno: um modelo de autoorganização? Rev Latino-am Enfermagem. 2006;14(3):44856. Disponível em: http://www.scielo.br/pdf/rlae/v14n3/ v14n3a21.pdf.

12. Canguilhem G. Novas reflexões referentes ao normal e ao patológico. Rio de Janeiro: Forense Universitária; 1963.

13. Aguiar A. Tomada de responsabilidade no campo da reforma psiquiátrica. In: Albuquerque P, Libério M, organizadoras. O cuidado em saúde mental: ética, clínica e política. Rio de Janeiro: Coordenação de Saúde Mental da Secretaria Municipal de Saúde do Rio de Janeiro; 2004. p. 37-44.
14. Saraceno B. Reabilitação Psicossocial: uma prática à espera de teoria. In: Pitta AMF, organizadora. Reabilitação psicossocial no Brasil. São Paulo: Hucitec; 1996. p.150-4.

15. Campos GWS, Amaral MA. A clínica ampliada e compartilhada, a gestão democrática e redes de atenção como referenciais teórico-operacionais para a reforma do hospital. Cien Saúde Coletiva. 2007;12(4):849-59.

16. Lima EA. Oficinas e outros dispositivos para uma clínica atravessada pela criação. In: Costa CM, Figueiredo AC, organizadoras. Oficinas terapêuticas em saúde mental: sujeito, produção e cidadania. Rio de Janeiro: Contra Capa; 2004. p.59-81.

17. Ribeiro MC. A saúde mental em Alagoas: trajetória da construção de um novo cuidado [tese]. São Paulo: Universidade de São Paulo; 2012 [citado 8 set. 2014]. Disponível em: http:// www.teses.usp.br/teses/disponiveis/83/83131/tde-17042012112917/en.php.

18. Campos RO, Furtado JP, Passos, E, Benevides R. À guisa de conclusão: la clé des champs! In: Campos RO, Furtado JP, Passos, E, Benevides R, organizadores. Pesquisa avaliativa em saúde mental: desenho participativo e efeitos da narratividade. São Paulo: Aderaldo \& Rothschild; 2008. p.68-77.

19. Lappann-Botti NC, Labate RC. Oficinas em saúde mental: a representação dos usuários dos serviços de saúde mental. Texto Contexto Enferm. 2004;13(4):519-26. Disponível em: http://www.scielo.br/pdf/tce/v13n4/a03.pdf.

20. Guerra AMC. Oficinas em saúde mental: percurso de uma história, fundamentos de uma prática. In: Costa CM, Figueiredo AC, organizadoras. Oficinas terapêuticas em saúde mental: sujeito, produção e cidadania. Rio de Janeiro: Contra Capa; 2004. p.23-58.

21. Santos BS. Pela mão de Alice: o social e o político na pósmodernidade. 9a ed. São Paulo: Cortez; 2010.

22. Bezerra Junior B. O cuidado nos CAPS: os novos desafios. In: Albuquerque P, Libério M, organizadoras. O cuidado em saúde mental: ética, clínica e política. Rio de Janeiro: Coordenação de Saúde Mental, Secretaria Municipal de Saúde; 2004. p.3-11. 